\title{
Traditional Bonesetters and Contemporary Orthopaedic Fracture Care in a Developing Nation: Historical Aspects, Contemporary Status and Future Directions
}

\author{
Benedict U. Nwachukwu ${ }^{*}$, Ikechukwu C. Okwesili ${ }^{2}$, Mitchel B. Harris ${ }^{1,3}$ and Jeffrey N. Katz ${ }^{1,3,4}$ \\ ${ }^{1}$ Harvard Medical School, USA \\ ${ }^{2}$ National Orthopaedic Hospital, Enugu, Nigeria \\ ${ }^{3}$ Department of Orthopedic Surgery, Brigham and Women's Hospital, 75 Francis Street Boston, MA 02115, USA \\ ${ }^{4}$ Orthopedic and Arthritis Center for Outcomes Research, Departments of Epidemiology and Environmental Health, \\ Harvard School of Public Health, USA
}

\begin{abstract}
In developing nations such as Nigeria, where there is a shortage of surgeons formally trained in fracture care, many of the injured seek care from traditional bonesetters. We conducted a qualitative study of fracture care in two settings in Enugu, Nigeria: The National Orthopaedic Hospital Enugu (NOHE) and a traditional bonesetter practice. Primary assessment measures at the NOHE included evaluations of the structure and process of fracture care according to the Orthopaedic Trauma Association's Level 1 Trauma Center Requirements. Further, we conducted interviews of NOHE patients and hospital staff. We also observed fracture care at a traditional bonesetter practice. We observed the traditional care process and interviewed both bonesetters and patrons of the bonesetter practice.

Although the NOHE does not qualify for certification as a Level 1 Trauma Center; the hospital does provide quality care. Our observations suggest a tension between Western and indigenous musculoskeletal practices. We propose that bonesetters not only be taught certain injury management techniques but also be incorporated into the Nigerian healthcare scheme. Bonesetters fill a void created by the severe lack of surgeons and further; bonesetters are primarily located in rural areas where they best care for underserved communities. In an integrated scheme, bonesetters would manage fractures for which they can achieve acceptable outcomes, referring others to local hospitals. An integrated model of fracture care is applicable in all developing countries where bonesetters perform a large proportion of fracture care.
\end{abstract}

Keywords: Traditional bonesetters, international orthopaedics, non-orthodox care.

\section{INTRODUCTION}

While treatment of illness has been woven into human history for millennia, in many developing countries Western medical concepts and practices have only appeared in the last 50 years. Not surprisingly, the use of traditional medicine in these developing countries remains widespread. For example, traditional medicine accounts for around $40 \%$ of all health care in China and up to $80 \%$ in Africa [1]. (We acknowledge that "traditional" depends on perspective; we adopt the convention used in the literature on indigenous practitioners, which refers to them as "traditional.") The popularity of traditional medicine is explained by a number of factors including availability, affordability, familiarity and custom. Many developing nations have integrated traditional practitioners into mainstream healthcare. For example, prenatal and birthing attendants, chiropractors and herbal practitioners have each found places in established healthcare schemes $[2,3]$.

*Address correspondence to this author at the Harvard Medical School, Holmes Society, 260 Longwood Avenue, $2^{\text {nd }}$ Floor, Boston, Massachusetts, MA 02115, USA; Tel: 617-732 5510; Fax: 617- 525 7900;

E-mail: Benedict_Nwachukwu@hms.harvard.edu
The use of traditional bonesetters to treat musculoskeletal injuries is also widespread in developing nations, particularly in Africa, Asia and South America [4-8]. In Nigeria, traditional bonesetters provide from $70-90 \%$ of the fracture care in certain areas [9]. The coexistence of traditional bonesetters and orthopedic care for fractures in Nigeria provides an opportunity to learn about the potential strengths and limitations of each method and to examine opportunities for cultural synthesis and collaboration.

To investigate these issues, we conducted a study of fracture care in Enugu, Nigeria. The aims of our investigation were to assess the quality of fracture care at a major tertiary care center in a developing nation; to better understand the impact of traditional bone setting on the practice of contemporary western Orthopaedics in that environment; and, to gather insights to develop a model of fruitful integration of traditional and Western fracture care.

\section{Background}

Nigeria is a regional power in Sub-Saharan African with vast wealth in natural resources, in particular petroleum. Nigeria's population is estimated to exceed 140 million, making it the eighth most densely populated country in the world and the most densely populated Black country [10]. 
However, despite the wealth of resources, the Nigerian population is plagued with poverty and an exceedingly high burden of disease (see Table 1). Musculoskeletal disorders constitute a large part of this burden.

Table 1. Baseline Characteristics of Nigeria compared to the United States*

\begin{tabular}{|c|c|c|}
\hline & United States & Nigeria \\
\hline \hline Population & $302,841,000$ & $144,720,000$ \\
\hline Gross national income per capita (\$) & 44,070 & 1,410 \\
\hline Life Expectancy at birth m/f (years) & $75 / 80$ & $48 / 49$ \\
\hline $\begin{array}{c}\text { Probability of dying under 5 } \\
\text { (per 1000 live births) }\end{array}$ & 8 & 191 \\
\hline Per Capita Health Expenditure (\$) & 6,714 & 50 \\
\hline $\begin{array}{c}\text { Total expenditure on health } \\
\text { as \% of GDP (2006) }\end{array}$ & 15.3 & 4.1 \\
\hline Physician Density (per 1000) & 2.56 & 0.28 \\
\hline $\begin{array}{c}\text { Orthopaedic Surgeon } \\
\text { Density (per 100,000) }\end{array}$ & 6.06 & 0.14 \\
\hline
\end{tabular}

*Information extracted from World Health Organization Country Profile: Nigeria and United States.

Nigeria currently has three major tertiary care centers devoted to Orthopaedics. In 1943 British colonials opened the first center in Igbobi Lagos as a rehabilitation camp for wounded soldiers returning to Nigeria from World War II. The hospital was named the National Orthopaedic HospitalIgbobi. Subsequently, two more centers have been established: The National Orthopaedic Hospitals in Kano and Enugu [11-13]. Nigeria has fewer than 200 orthopaedic surgeons. While the number of traditional bonesetters is not accurately documented, it is widely accepted that traditional fracture care is utilized more than westernized orthopaedic services.

\section{METHODS}

We used a qualitative research design to investigate the roles and interplay between traditional fracture care and contemporary western Orthopaedics in a developing nation. Qualitative strategies do not test hypotheses based on statistical significance and therefore usually do not report quantitative findings as sampling of subjects may be nonrepresentative. However, qualitative strategies do allow investigators to collect rich narrative data, gain insight into the perspectives of study respondents and generate new hypotheses for further quantitative research $[14,15]$.

We critically appraised services provided at National Orthopaedic Hospital Enugu (NOHE) using the Orthopaedic Trauma Association's criteria for certification as a Level I trauma center [16] (Compared to the Orthopaedic Trauma Association's criteria, the American College of Surgeon's trauma certification criteria are more widely implemented in the U.S.; however we chose to use the former criteria as they are more applicable to trauma centers in developing nations. Further, our goal was not to compare fracture care in Nigeria with U.S. standards, but rather to analyze fracture care in Nigeria using an internationally accepted minimum set of standards). We recruited and interviewed orthopaedic surgeons assigned to the NOHE's trauma service; we interviewed them using a standardized questionnaire. We also interviewed a series of patients using a standardized questionnaire.

We assessed fracture care at a bone setting facility in Anambra State (an adjacent state to Enugu State where the NOHE is located). We documented the fracture care provided by the traditional bonesetters over the course of three days and we conducted semi-structured interviews of the two chief bonesetters as well as patients in the bone setting practice. All interviews both at the NOHE and at the bonesetter facility were conducted by two authors (BUN, ICO).

This study was approved by the Committee for Human Subjects Research at Harvard Medical School as well as the Ethics Committee at the National Orthopaedic Hospital Enugu.

\section{FINDINGS}

\section{National Orthopaedic Hospital Enugu (NOHE)}

The NOHE is the major trauma center in Southern Nigeria and is located in Enugu State (population 5,590,513). Although the NOHE is located in an urban setting, the hospital receives the majority of its patients from the surrounding rural areas. The NOHE has a 220-bed capacity and an annual in-patient volume of more than 30,000 patients.

We analyzed fracture care at the NOHE using the suggested requirements for a Level I Orthopaedic Trauma Association Trauma Center [16]. We found that the NOHE met these criteria in some areas and but not in others (Table 2). An area of particular deficiency was Support Service. The NOHE does not have other subspecialty surgeons on staff beside Orthopaedic and Plastic surgeons. The hospital also lacks several on-site diagnostic services such as MRI, CT and angiography. However for the most part, the NOHE attains several other standards comparable to major trauma centers in the U.S. We present a complete listing of our assessment findings in Table 2.

A total of six orthopaedic surgeons were interviewed for this study. All six surgeons were born and received their medical training in Nigeria. Further, they all spoke the major dialect of the region- Igbo. Three of the six surgeons were fully trained and licensed while the other three were senior residents in NOHE's seven year residency program. There were no female surgeons assigned to the trauma service at the time of this study; therefore, all six interviewed surgeons were male. All six confirmed that prior use of traditional bonesetters was common among patients hospitalized for fractures and that bone-setting practices were ubiquitous in the region. However none of the surgeons had personally observed musculoskeletal care at a bonesetter practice. When asked: "What are some of the difficulties you face in achieving optimal patient results?" all six surgeons identified bone setting practices as an obstacle to their work at the NOHE. One of the surgeons explained that patients present to the NOHE late, often when traditional treatment has failed to achieve acceptable results. At this point the burden of disease is advanced, increasing the complexity of cases as well as the risk of complications. Secondly, patients present 
Table 2. Comparison of the National Orthopaedic Hospital Enugu (NOHE) according to the Orthopaedic Trauma Association's (OTA) minimum standards for a Level I Trauma Center*

\begin{tabular}{|c|c|}
\hline OTA Mandate & NOHE Status \\
\hline \multicolumn{2}{|l|}{$\underline{\text { Staffing }}$} \\
\hline \multicolumn{2}{|l|}{ A) Trauma Director who: } \\
\hline i) Is adequately trained in orthopedic Trauma & YES \\
\hline ii) Actively participates in trauma call & YES \\
\hline iii) Acts as a liason to Trauma team and has authority to correct deficiencies in trauma care & YES \\
\hline C) Appropriate number of mid-level staff in hospital system including cast/traction technicians, trauma dedicated nursing team & YES \\
\hline \multicolumn{2}{|l|}{$\underline{\text { Support Services }}$} \\
\hline \multicolumn{2}{|l|}{ A) Physician specialists: } \\
\hline iv) Neurosurgeon & NO \\
\hline v) Spine Surgeon (Orthopaedic or neurosurgeon) & YES \\
\hline vi) Medical specialists available for infectious disease, cardiac, pulmonary, dermatologic, `’ psychiatric and rehabilitation consult & NO \\
\hline B) MRI, CT, and angiography suites available in house and 24/7/365. & NO \\
\hline \multicolumn{2}{|l|}{ Operating Room Equipment } \\
\hline Long bone inntramedullary nailing system & YES \\
\hline Ex fix system (mini, small, large, pelvic) & YES \\
\hline Plating system (mini, small, large, locked and standard) & YES \\
\hline Hip fracture fixation system (plates and nails) & YES \\
\hline Screw removal sets & YES \\
\hline Arthroscopy system** & YES \\
\hline General Orthopaedic tools which are dedicated to trauma service and not shared with other orthopaedic services & NO \\
\hline
\end{tabular}

*Table 2 contains only a sampling of major OTA requirements. Some of the requirements omitted in this table were either satisfied by the NOHE or were considered inapplicable in the context of the NOHE. Please refer to the OTA website for a full listing of requirements

**These items are present at the NOHE but are infrequently used in the trauma context. During the period of evaluation, these items were not used.

to the NOHE with an innate distrust of orthodox fracture care. The patient distrust complicates the surgeon's work because the patient is more likely to be non-compliant and resistant to care paths.

A total of eight patients were interviewed for this study. All eight patients had received surgery at the hospital and were in the process of post-operative care. Five of the eight interviewed patients stated that they had sought traditional fracture care with a bonesetter before reporting to the NOHE. Of these five patients initially treated by a bonesetter, two subsequently required amputation. Of the five patients who had sought traditional care before reporting to the NOHE, all five had prior knowledge of the NOHE; however they selected the bonesetter for a variety of reasons including lack of orthopaedic care availability, personal preference and fear of invasive procedures. All interviewed patients expressed satisfaction with their level of care at the NOHE; however several were concerned about their future and the speed of their recovery.

\section{Traditional Bonesetter}

We identified and visited a bone setting practice in Anambra Nigeria, less than 40 miles from the NOHE. The bonesetters operated in a large domestic compound located in a rural community. Two chief bonesetters managed the clinic (mother and son) with the aid of support staff. During our stay, the traditional clinic had over 50 patients residing on grounds (Fig. 1). 
(A)

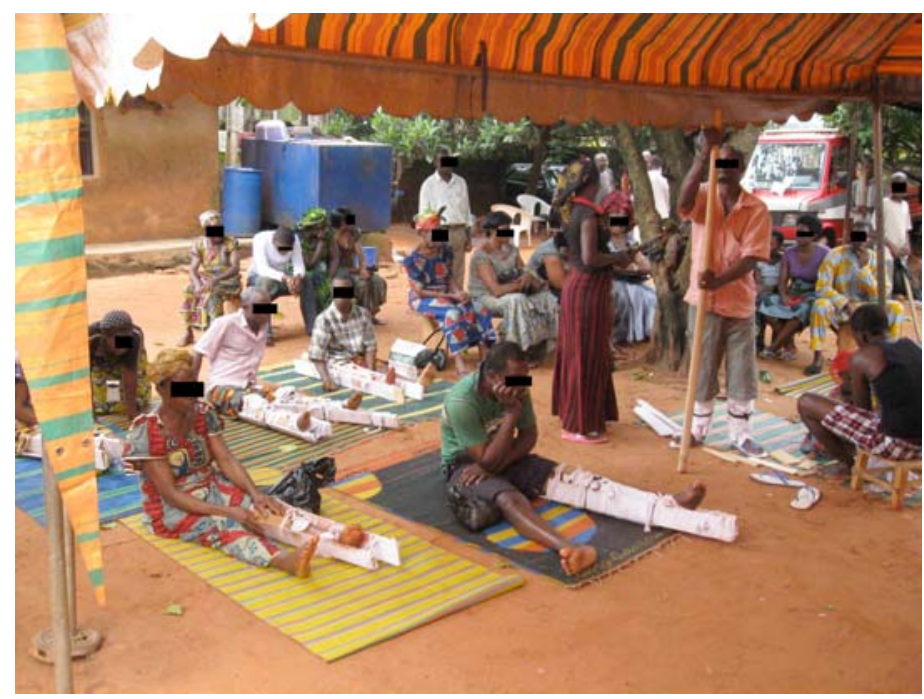

(B)

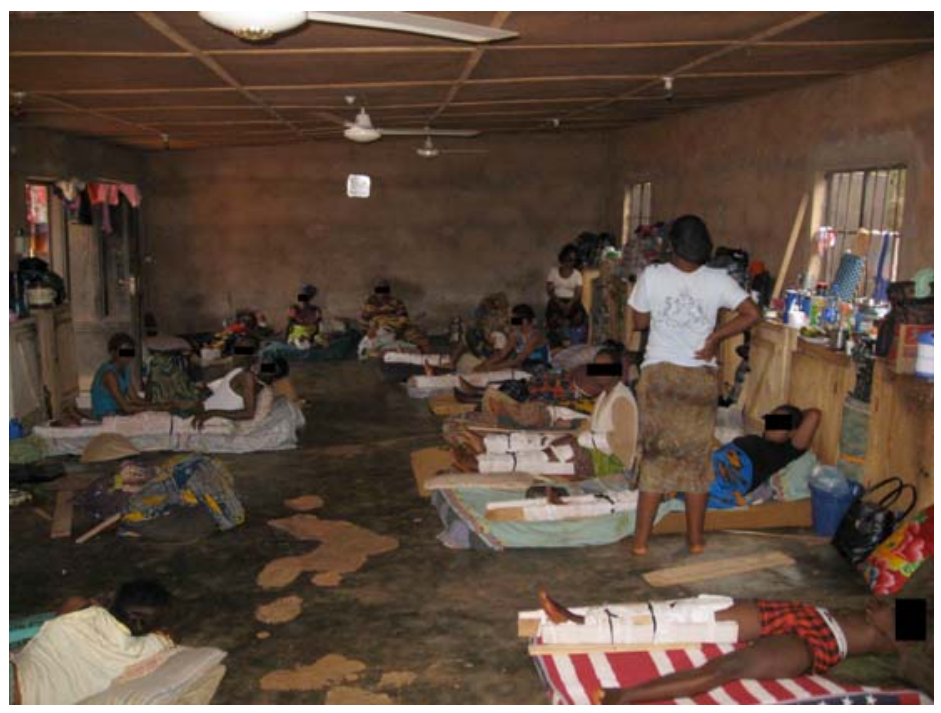

(C)

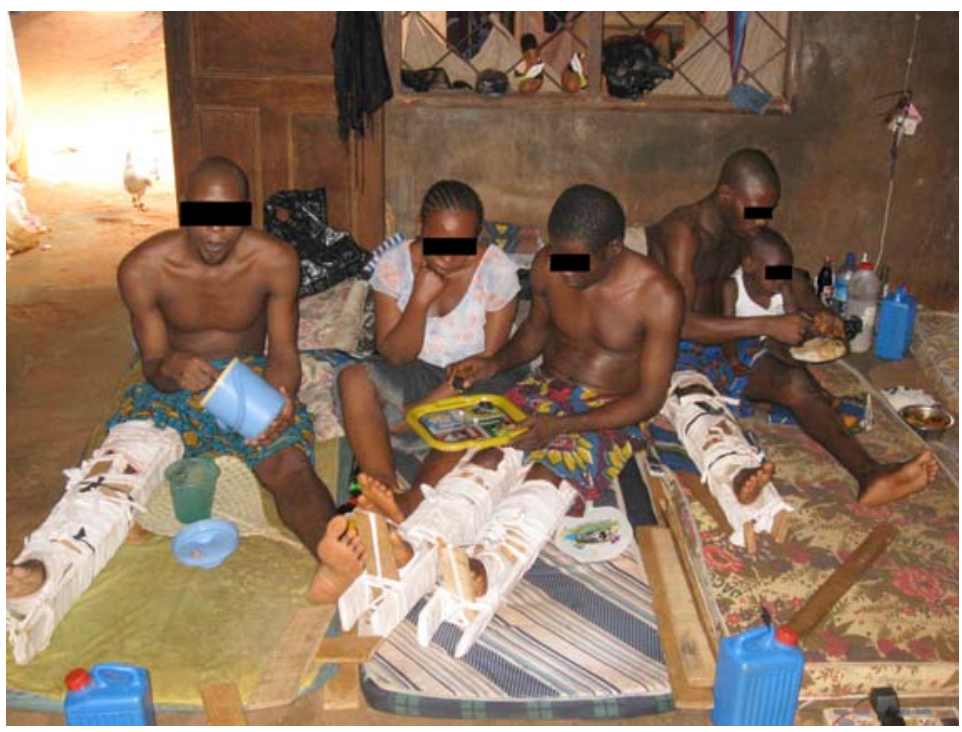

Fig. (1A). (A) Traditional bonesetter patrons waiting for care. (B, C) Traditional bonesetter patrons in their on-site living accommodations. 


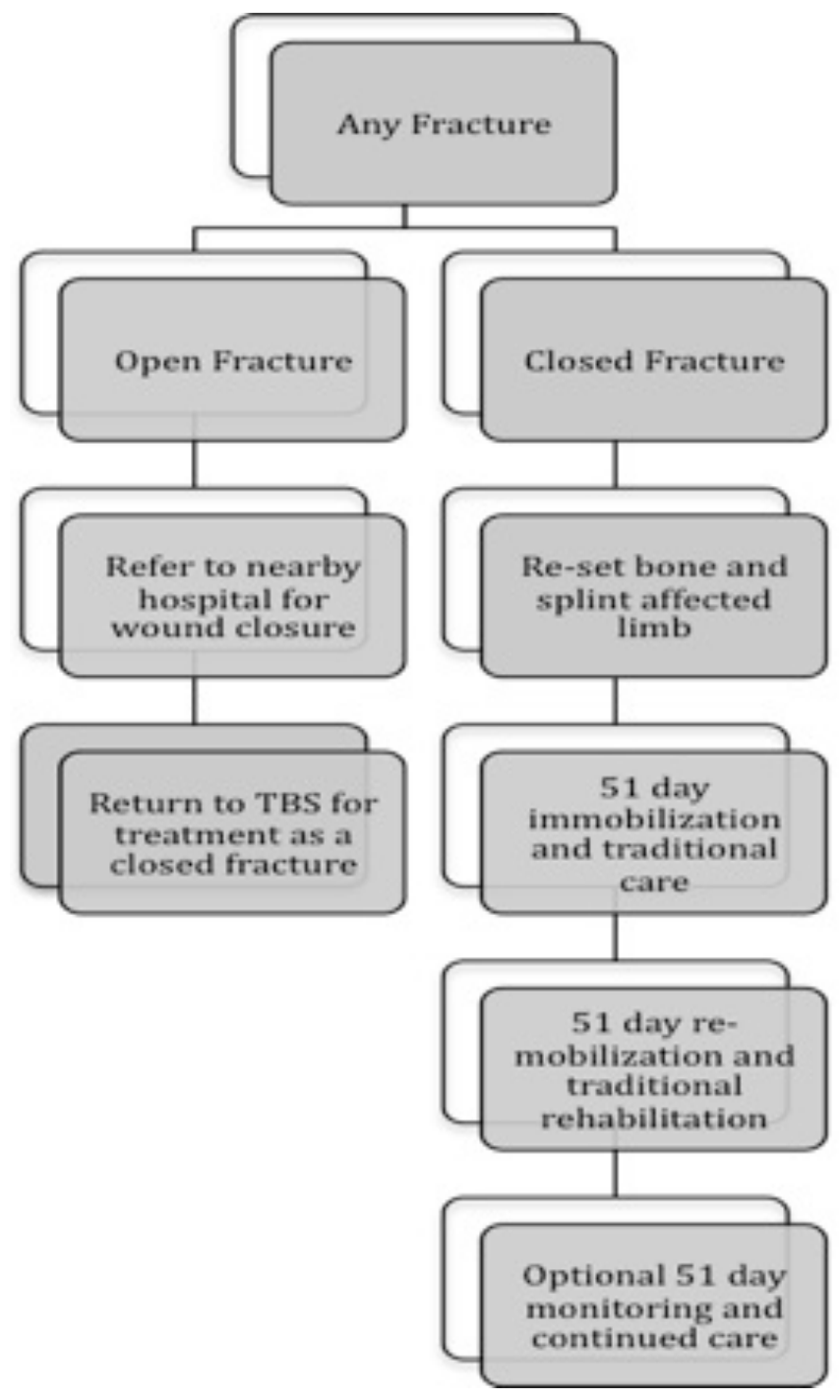

Fig. (2). Observed Traditional bonesetter care path.

The care processes and treatment of fractures at the traditional bonesetter are outlined in Fig. (2). The observed care path was the same for both lower and upper extremity fractures. Further, the same care path was applied to children, adolescents and adults. The first step in the traditional bonesetter treatment algorithm is to identify whether a fracture is open or closed; the bonesetters refer open fractures to a local clinic for wound care and closure. Upon the patients' return to the bonesetters, the limb is manipulated and treated as a closed fracture. For closed fractures, the bonesetters identify the fracture site using palpation and clinical signs. Once the fracture site has been identified, the bonesetters attempt to reduce the fracture to its anatomical position. Following reduction, the bonesetters apply an herbal cream (known as "Ufie") to the affected limb while delivering an incantation.

After reduction and embalming, the affected limb is splinted to prevent limb movement. For lower extremity fractures, weight bearing on the affected limb is prohibited. Splinting materials include cloths, hard cardboard and plywood. Once the limb is splinted, the patient is begun on a standard care pathway, which involves 51 days of complete limb immobilization followed by a 51-day period of rehabilitation and return to function. During the immobilization period, the splinting materials are changed every four days, at which point the traditional bonesetter reapplies the herbal cream and massages the limb. For patients with lower extremity fractures, during the immobilization period, the patient is given a personal mat on which they can be carried around by family members or support staff. During the second 51 days, patients are gradually mobilized and the bonesetter continues to massage and embalm the limb weekly while also counseling the individual on gait training. The care process in the final 51 days is directed at the discretion of the bonesetter based on the signs and symptoms of the patient.

Our interview of the bonesetters revealed that neither bonesetter had "formal" training in fracture care. The elder bonesetter learned her craft as an apprentice to another bonesetter and she passed on the training to her son. We interviewed both bonesetters at length and we surveyed twelve bone setting patients to uncover reasons for the widespread use of bonesetters. Their responses suggested five key reasons: 1) Fear of implants and foreign objects including musculoskeletal traction devices; 2) Belief in the spiritual powers of the bonesetter; 3) Convenience and flexibility of traditional care setting; 4) Prohibitive cost of orthopaedic care in a hospital setting; 5) Familiarity with bonesetter culture and lack of familiarity with orthopedic centers. One of the bonesetters explained that she even counsels her patients and their families to avoid Orthopaedic centers because many of those who use such facilities "return with missing legs or with metal in their body" [loosely translated from the original Igbo].

\section{DISCUSSION}

Our study draws on perspectives of both orthodox and indigenous/traditional practitioners. We interacted with the injured as they received orthodox and traditional musculoskeletal care. Our study supports the observations of others [5-9] that although contemporary fracture care is available in Nigeria, the traditional bonesetter is the practitioner of choice for many Nigerians with fractures.

A historical perspective is appropriate in order to provide the reader with better insight into the tension between traditional and contemporary care in Nigeria and many other developing nations. Traditional bonesetters have had roots in most countries' early cultural history [17]. Traditional bonesetting arose as an adaptive approach to injury care. With the advent of new technologies and advancements in medicine, traditional fracture care evolved into what we recognize today as contemporary orthopaedics. However in developing nations where advances in medical technology have been disjointed and non-equally distributed, the traditional and contemporary approaches have been forced into a disharmonious coexistence.

A closer examination of the Nigerian healthcare system sheds some light on why it has been particularly difficult to integrate contemporary western Orthopaedics with indigenous practices. The first hospital in Nigeria was established in 1873. However, that hospital was established primarily by the European Colonials with the intent to primarily treat "their own". It took another 40 years until Nigerians could actually use that hospital, and even then, only select elite Nigerians were welcomed. Similarly, when 
the National Orthopaedic Hospitals were first established, they were intended solely for Europeans. Nigerian physicians only began to receive training in Orthopaedics after the country's Independence from Great Britain in 1960. Thus, while the Nigerian populace at large has been using the western medical approach for only a matter of decades; they have been using traditional methods for millennia. Naturally, integration of the traditional and contemporary approaches will be slow and deliberate, especially because of the contemporary approach's association with an oppressive colonial past.

\section{An Integrated Approach}

A collaborative effort on the part of governments, professional orthopaedic societies, private/charitable organizations and traditional healers is needed to integrate modern fracture care in developing nations. We support the notion that further integration between traditional and western practices will ultimately provide sustained long-term improvement of outcomes after musculoskeletal injuries.

The first step toward integration is to appoint an impartial third party organization charged by the Nigerian healthcare system to bring the two cultures together. The third party organization should appeal to prominent figures within the traditional and orthodox groups. The third party must then bring these individuals together enabling them to identify common goals and to understand the possible roles for their distinct approaches in an integrated scheme. One of the key tasks for the third party mediator will be to objectively present data on complications, function and satisfaction from each of the care-providers' practices. The third party must convey to bonesetters data on complications while also encouraging the orthodox surgeons to learn from the bonesetters why traditional techniques have maintained such high popularity and patient satisfaction.

Once widespread understanding has been reached between bonesetters and orthopedic surgeons, local and regional hospitals can then begin implementing training programs. It is important however that these programs not seek to eradicate the fundamentals of traditional practices, but rather introduce compatible elements of contemporary fracture care. For example, all bonesetters should be taught to refer open wounds to local hospitals. Although the bonesetters whom we observed followed this algorithm, many do not. Significant complications can arise from bonesetters manipulating open wounds. If resources allow, the use of radiography could be introduced to aid bonesetters in their diagnosis and care. The overarching goal of any bonesetter-training program should be that the bonesetters understand which fracture types to treat and which fracture types to refer to the hospital. Under this model, the traditional bonesetter continues to serve as the primary point of contact for many patients. However, through collaborative referrals and safer practices, better outcomes will be achieved as complex patients present to orthopaedic centers earlier and with less apprehension.

Such an integrated healthcare scheme is not without major obstacles. Key challenges include costs of care, organizational complexity and threats to each group's referral base and to the status quo. We suggest that these problems are likely tractable and certainly worthy of confronting given the frequency of fracture and the high costs of fracture care and its complications.

\section{CONCLUSION}

Our African experience documented the cultural clash between traditional and western approaches to fracture care in Nigeria. Even though contemporary orthopaedic trauma care in Nigeria meets many standards for trauma care in the US, the majority of fracture victims initially visit a traditional bonesetter, only presenting to the hospital if and when serious complications arise. The available literature suggests that this trend is present in many developing nations throughout Africa, Asia and South America [4, 18, 19]. We propose that Orthopaedic surgeons and bonesetters work collaboratively. The first step toward integration involves undertaking studies to better understand the morbidities associated with bone setting care as well as the types of injuries that bonesetters typically handle proficiently. Informed by this knowledge, healthcare policy makers can develop a fracture care scheme in which bonesetters manage fractures for which they can achieve acceptable outcomes, referring others to local or regional hospitals. Such an integrated scheme will benefit patients, orthopaedic surgeons and bonesetters alike in developing nations. Patients will receive culturally compatible, streamlined care with fewer complications, while physicians and bonesetters will be able to address the burden of fractures in developing countries with an optimal deployment of culturally compatible care and technical expertise.

\section{ACKNOWLEDGEMENTS}

This study was approved by the Committee for Human Subjects Research at Harvard Medical School as well as the Ethics Committee at the National Orthopaedic Hospital Enugu.

This work was primarily performed in Enugu, Nigeria at the National Orthopaedic Hospital Enugu.

The Authors have no financial relationships or commercial associations that might pose a potential conflict of interest in connection with this work.

\section{REFERENCES}

[1] World Health Organization. WHO Traditional Medicine Strategy 2002-2005. Geneva, World Health Organization 2002.

[2] World Health Organization. Consultation Meeting on Traditional Medicine and Modern Medicine: Harmonizing the Two Approaches. Geneva, World Health Organization 1999 (document reference (WP)TM/ICP/TM/001/RB/98-RS/99/GE/32(CHN)).

[3] World Health Organization. Promoting the Role of Traditional Medicine in Health Systems: a Stategy for the African Region 2001-2010. Harare, World Health Organization 2000 (document reference AFR/RC50/Doc.9/R).

[4] Aries MJH, Joosten H, Wegdam HHJ, van der Geest S. Fracture treatment by bonesetters in central Ghana: patients explain their choices and experiences. Trop Med Int Health 2007; 12(4): 564-74.

[5] Onuminya JE. The role of the traditional bonesetter in primary fracture care in Nigeria. S Afr Med J 2004; 94(8): 652-8.

[6] Thanni LOA. Factors influencing patronage of traditional bone setters. West Afr J Med 2000; 19(3): 220-4.

[7] Nwadiaro HC, Ozoilo KN, Nwadiaro PO, Kidmas AT, Oboiren M. Determinants of patronage of traditional bone setters in the middle belt of Nigeria. Niger J Med 2008; 17(3): 356-9.

[8] Nwankwo OE, katchy AU. Limb gangrene following treatment of limb injury by traditional bone setter (Tbs): a report of 15 consecutive cases. Niger Postgrad Med J 2005; 12(1): 57-60. 
[9] Omololu AB, Ogunlade SO, Gopaldasani VK. The practice of traditional bonesetting: Training algorithm. Clin Orthop Relat Res 2008; 466: 2392-8.

[10] Country Profile: Nigeria. BBC News 2008-04-30. (Accessed at http://news.bbc.co.uk/2/hi/africa/country_profiles/1064557.stm).

[11] National Orthopaedic Hospital, Igbobi, Lagos, Nigeria: About Us (Accessed at http://orthopaedicigbobinigeria.org).

[12] National Orthopaedic Hospital, Dala-Kano, Nigeria: History of NOH, Dala-Kano (Accessed at http://www.orthopaedicdala.org/).

[13] National Orthopaedic Hospital Enugu (Accessed at http://www.nohenig.com/background.htm).

[14] Sofaer S. Qualitative research methods. Int J Qual Health Care 2002; 14: 329-36.
[15] Barbour RS. The role of qualitative research in broadening the 'evidence base' for clinical practice. J Eval Clin Pract 2000; 6(2): 155-63.

[16] Orthopaedic Trauma Association. Minimum Requirements for Certification as an OTA Trauma Center (level I). Accessed at: http://www.ota.org/policy/C2\%20Action\%20Trauma\%20center\%2 0criteria\%20-\%20revised\%208-18\%20anglen2.pdf.

[17] Agarwal A, Agarwal R. The practice and tradition of bonesetting. Education Health (Abingdon) 2010; 23(1): 225.

[18] Banskota AK. Musculoskeletal training for orthopaedists and nonorthopaedists: experiences in Nepal. Clin Orthop Relat Res 2008; 466(10): 2369-76.

[19] Anderson R. The treatment of musculoskeletal disorders by a Mexican bonesetter (sobador). Soc Sci Med 1987; 24(1): 43-6.

Received: May 31, 2010

Revised: September 8, 2010

Accepted: September 25, 2010

(C) Nwachukwu et al.; Licensee Bentham Open.

This is an open access article licensed under the terms of the Creative Commons Attribution Non-Commercial License (http://creativecommons.org/licenses/by$\mathrm{nc} / 3.0 /$ ) which permits unrestricted, non-commercial use, distribution and reproduction in any medium, provided the work is properly cited. 